Health and Medicine | The Human Vascular Research Group (HVRG)

\section{Clinical relevance aims to uncover novel regulators of human coronary artery function}

\begin{tabular}{|c|c|}
\hline $\begin{array}{l}\text { Charting new territories in } \\
\text { vascular disease research, The } \\
\text { Human Vascular Research Group } \\
\text { (HVRG) at the Medical College } \\
\text { of Wisconsin uses fresh human } \\
\text { tissues to address important } \\
\text { questions in vascular biology. } \\
\text { Focusing on the microcirculation, } \\
\text { the smallest of blood vessels } \\
\text { inside our bodies, their aim is } \\
\text { to better understand coronary } \\
\text { artery disease (CAD) and other } \\
\text { clinical problems associated with } \\
\text { microvascular defects. Heart } \\
\text { disease is the leading cause } \\
\text { of death worldwide and new } \\
\text { approaches to treatment } \\
\text { and prevention are vital. }\end{array}$ & $\begin{array}{l}\text { he microcirculation is made up } \\
\text { of a network of blood vessels, } \\
\text { including small arterioles, } \\
\text { capillaries, and venules. Its primary } \\
\text { function is to supply oxygen and nutrients } \\
\text { to tissues and remove metabolic waste. } \\
\text { The microcirculation is responsible for } \\
\text { orchestrating adjustments of blood } \\
\text { vessels to match local blood supply with } \\
\text { oxygen demand of tissues - both in } \\
\text { normal resting conditions and in response } \\
\text { to external stimuli (e.g., increased heart } \\
\text { rate) that require increased amounts } \\
\text { of oxygen and nutrients. } \\
\text { THE MICROCIRCULATION AND } \\
\text { CORONARY ARTERY DISEASE } \\
\text { Heart disease is the leading cause of } \\
\text { death worldwide and a significant portion } \\
\text { of heart-related deaths are directly linked } \\
\text { to blood vessel dysfunction. Changes } \\
\text { in the microcirculation are a well-known } \\
\text { early indicator for coronary artery } \\
\text { disease (CAD). While age and onset } \\
\text { of CAD are associated with changes } \\
\text { in the requlation of dilation of human }\end{array}$ \\
\hline
\end{tabular}

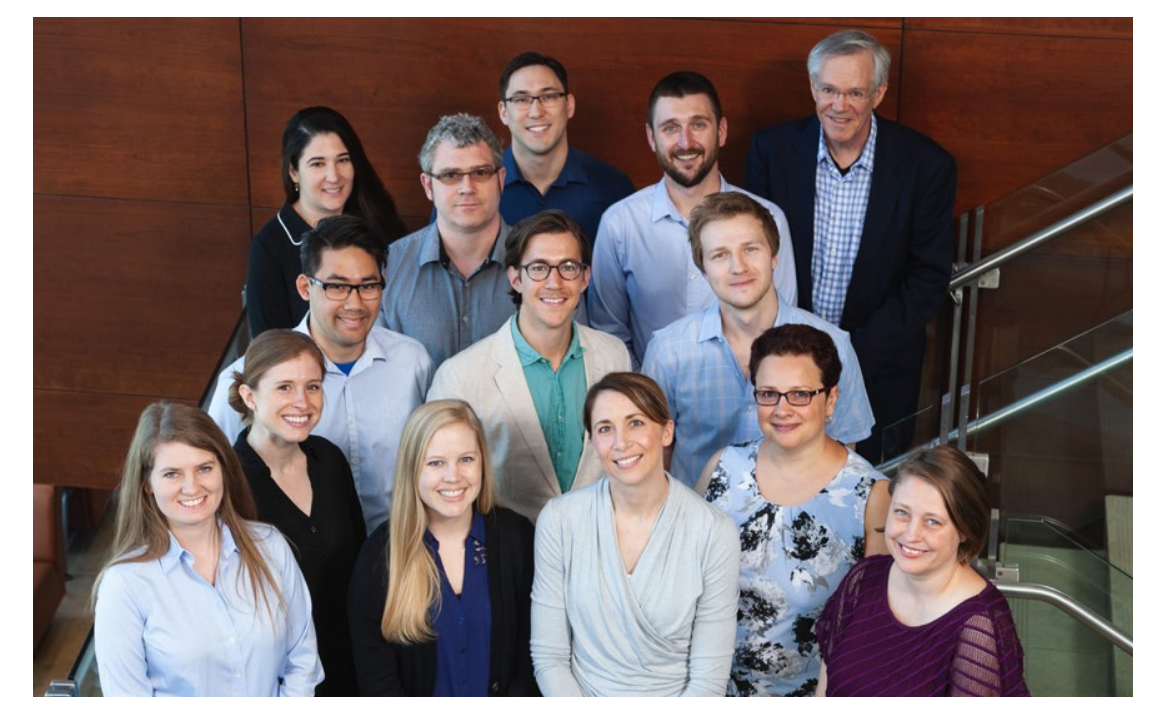

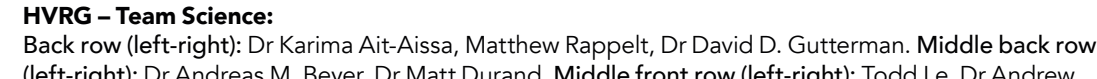

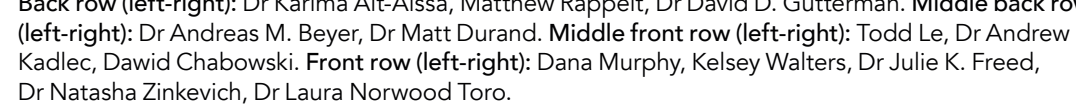

coronary and adipose microvessels, the factors that regulate these changes are poorly understood. Although animal models provide some insight into blood vessel regulation in health and disease, they often do not translate directly into humans.

As microvascular function is extremely challenging to measure directly in to interrogate blood vessel function of pathological changes observed in human subjects. Taking a unique approach, the Human Vascular Research Group (HVRG), consisting of Drs Julie Freed, David Gutterman, Matt Durand and Andreas Beyer, is set to increase our mechanistic understanding of changes observed with onset of disease. The group uses isolated microvessels from surgically discarded tissue to explore why microvascular dysfunction is a powerf predictor of cardiovascular disease, Their aim is to identify clinically relevant pathways and nivestigate these in more models in support of the findings obtained from human microvessels. Their hope is to uncover novel targets for treatment and prevention of human heart conditions.

ASSESSING MICROVASCULAR FUNCTION

The team has a wealth of expertise in human tissue samples, an approach that specialised method employs direct videomicroscopy of cannulated, pressurized arterioles to assess microvascular function in vitro (outside the body). Through this, the HVRG team has unearthed severa signalling pathways that play a key during normal conditions, acular tone and chronic disease that impact the are needed to further our understanding studying microcirculatory function in intact provides obvious clinical relevance. Their

CHANGE IN MEDIATORS OF FMD THROUGHOUT LIFE

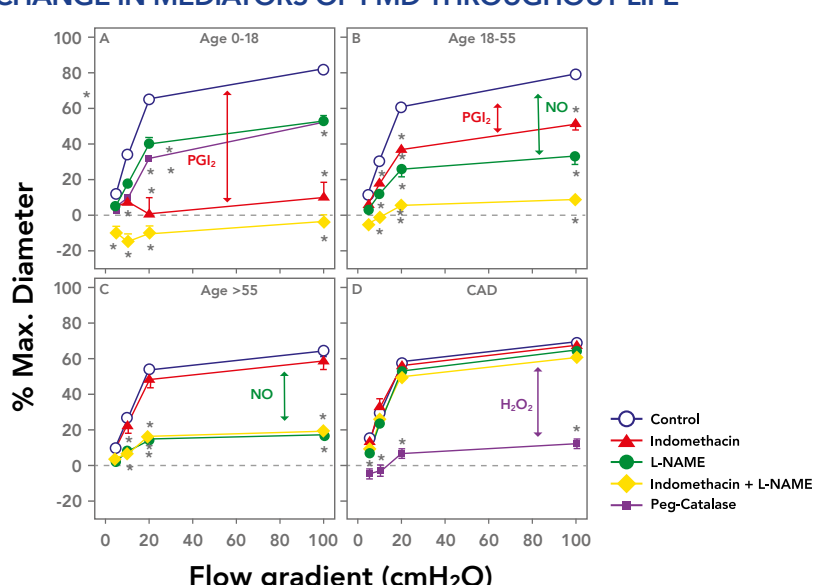

MECHANISMS OF ENDOTHELIUM-DEPENDENT DILATION

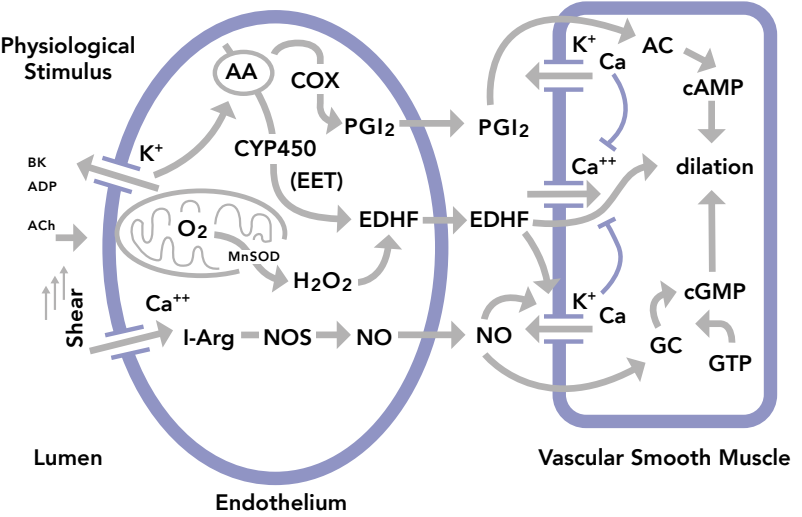

(2) onset of disease. Left Flow-mediated dilation (FMD) in isolated atrial

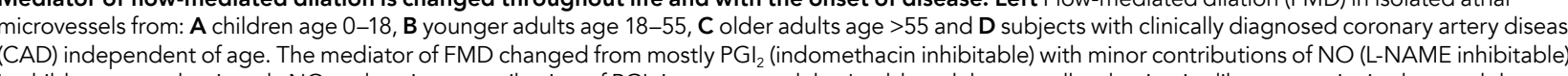

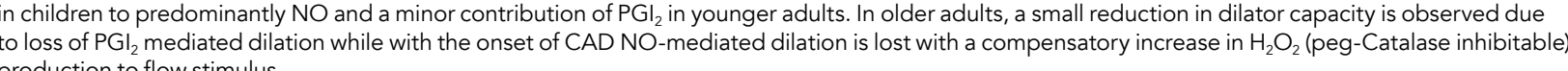

understanding and treatment of a variety of cardiovascular disorders.

\section{A NOVEL APPROACH}

The human microcirculation not only

plays a role in regulating tissue perfusion of the local tissue environment. The team believes that this provides an explanation for the multitude of dilator and constrictor factors released from the endothelium (the cells lining the blood vessel walls) that also influence vessels further downstream and the function of surrounding tissues.

The HVRG team proposes a novel idea: that communication between microvessels and their surrounding of nitric oxide (NO, what is, he release The by blocking inflammation. Conversely, in disease states or stress conditions. the release of other substances from the endothelium leads to cellular dysfunction, thrombosis, and fibrosis. Their theory neatly explains why microvascular dysfunction is such powerful predictor of cardiovascular injury

Cardiovascular stress and disease expose the dynamic nature of the mediators of microvasculature dilation, which can be changed either acutely (e.g. by changes in pressure within the vess or pharmacological interventions) or by

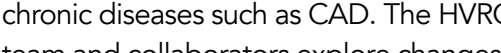
team and collaborators explore changes in the dilator pathways, hoping to
The team has a wealth of expertise in studying microcirculatory function in intact human tissue samples, an approach that provides obvious clinical relevance.

provoked dysfunction leads to clinical implications (e.g. atherosclerosis).

\section{THE STRESS-INDUCED SHIFT IN} MEDIATOR OF VASODILATION In human coronary arterioles from patie with CAD, loss of nitric oxide-mediated dilation to shear stress flow-mediated dilation, FMD) is compensated by the release of hydrogen peroxide $\left(\mathrm{H}_{2} \mathrm{O}_{2}\right)$ from mitochondria in the endothelia cells. Interestingly, both hydrogen peroxide and nitric oxide are vasodil the health of the microvasculature and surrounding tissue - nitric oxide promotes latency whereas $\mathrm{H}_{2} \mathrm{O}_{2}$ prom inflammation and atherosclerosis. The HVRG is exploring the mechanisms tha switch the mediator released from the endothelium, believing it to be pivotal in reducing tissue stress from inflammation. The researchers are investigating adaptive autophagy (the regulated mechanism components), which is shown to be necessary for the endothelial shearinduced production of nitric oxide. Their hypothesis is that by blocking autophag lux, NO is reduced, and inflammation NO pred, leading to a change from $\mathrm{NO}$ production to $\mathrm{H}_{2} \mathrm{O}_{2}$ as the mediator
A PIVOTAL ROLE OF ENDOTHELIAL MITOCHONDRIAL FUNCTION

Two pathways involving the production activity are critical in the shift from NO to $\mathrm{H}_{2} \mathrm{O}_{2}$. Both ceramide and reduced thich mitochond function and promote $\mathrm{S}_{2} \mathrm{O}_{2}$ Thel free radical production such is to protect the nunal role of telomerase hortening duncear genome from arepeat sequince to telomes regions of repetitive DNA sequences at the end of chromosomes); thus telomerase TERT, the catalytic subunit of telomerase is translocated to the mitochondria where it has been shown to bind to mitochondria DNA (mtDNA). The exact role of TERT in the mitochondria is somewhat puzzling as mtDNA is circular and does not have telomeres to protect. Ceramide, of sphingomyelinase is produced in endothelial cells, found in human plasma and is a risk factor for atherosclerosis. Ceramide is a known inhibitor of telomerase activity in the cancer literature, both active targets of forth active targets of investigation damage. Under conditions of acute stress, 
however, to date unsuccessful. One of the reasons why ceramide or anti-telomerase cancer treatment may be the general toxicity of such drugs that manifests as cardiovascular toxicity On the other hand, however, targeting ceramide and telomerase or their associated pathways may be a novel therapeutic strategy for the treatment of coronary artery disease as well as cancer if risk versus benefit analysis is carefully evaluated.

As mitochondrial function appears to be critical for physiological vasodilator capacity in the human heart, the HVRG is investigating underlying mitochondrial damage as the causative link betwe these pathways. Both activation of telomerase or inhibition of ceramide production contribut so the prevention demonstrated that TERT $r$ activation and translocation to the mitochondria, as well as decreasing levels of ceramide, can restore the "normal" mechanism of flowinduced endothelium-dependent dilation from $\mathrm{H}_{2} \mathrm{O}_{2}$ to $\mathrm{NO}$ (thus moving away from a CAD phenotype). Their central tenet is that mtDNA damage is one of the underlying causes that leads to an increase in ROS production. Mitochondria ROS has been shown to promote the development of arteriolosclerosis and endothelial dysfunction, predisposing individuals to vascular complications. At the same time, NO has a well-know

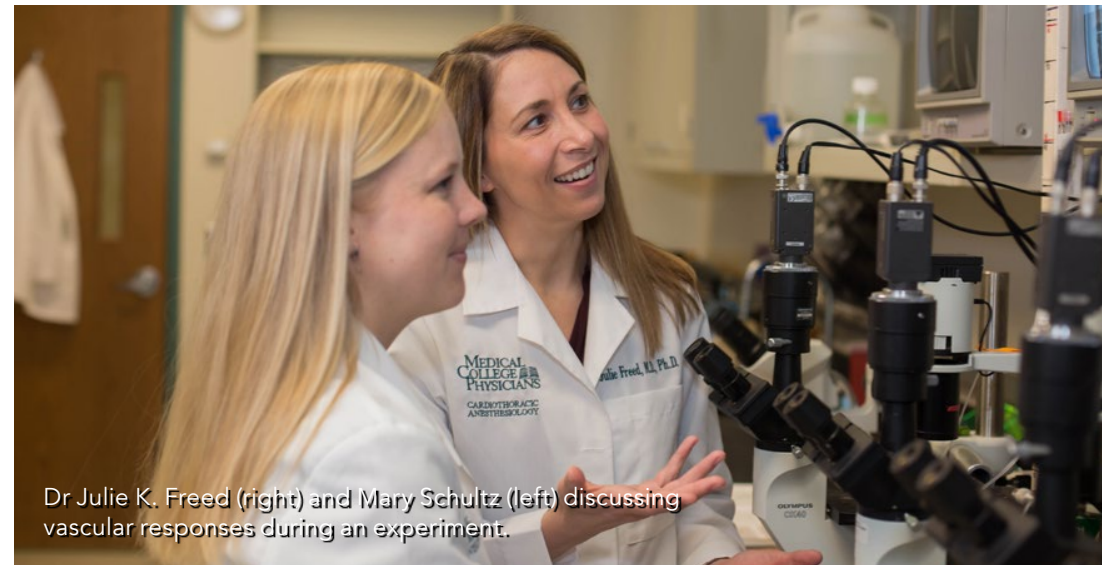

Their theory neatly explains why microvascular dysfunction is such a powerful predictor of cardiovascular injury. inhibitory effect on the generation to increase telomerase activity.

The relevance of these findings extends itself beyond the laboratony. Dr Durand in collaboration with Dr Allison Hyngstron at Marquette University, were the first investigators to quantify the hyperemic blood flow response to muscle contractions in the legs of stroke survivo They discovered that reduced blood flow to the paretic leg (impaired function as a result of a stroke) during muscle contraction was strongly correlated with reduced leg strength and poor motor

REGULATION OF THE MECHANISM OF FLOW INDUCED DILATION IN THE HUMAN MICROCIRCULATION

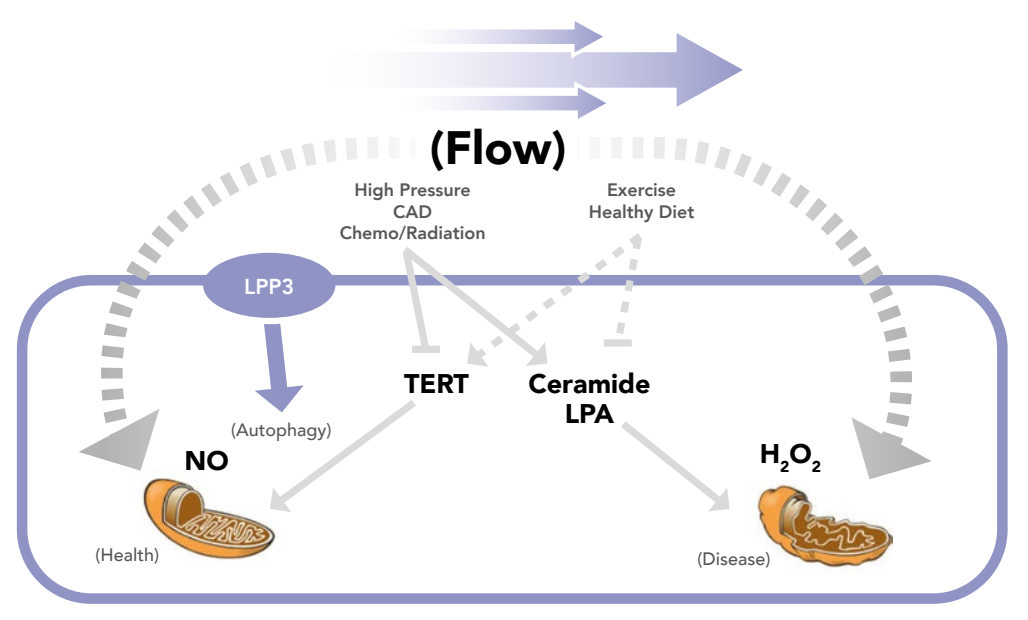

In healthy adult subjects (left), arteriolar endothelium produces NO which support a quiescent
nonproliferative/anti- atherosclerotic state. With onset of disease (CAD) or exposure to acute stress nonproliterative/ant-a atherosslerotic state. With onset of disease (AD) or exposure to acute stress
(increased intralumininal pressure), flow through the microvasculature releases hydrogen peroxide,

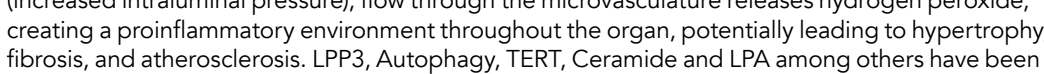
fibrosis, and atherosclerosis. LPP3, Autophagy, TERT, Ceramide and LPA among
identified to play important parts in regulating this switch in dilator mechanism. that the peripheral microcirculation (leg) is significantly impaired after a stroke and of motor function in this population. Further, the Durand lab is exploring simple non-invasive means to improve a technique called ischemic conditioning (short, intermittent bouts of limb ischemia using a blood pressure cuff is sufficient to improve paretic leg strength and hyperemic blood flow by approximately $15 \%$. This improvement in strength is also accompanied by an acute improvement Currently, Drs. Durand and Freed from the HVRG are jointly conducting a clinica trial which will examine the effects of vascular function and frailty in pre-surgical, vascular fonction and fraily in pre-surg traditionally have poor surgical outcomes.

Together these findings underline the growing recognition that dysfunction of the microcirculation contributes to the development of cardiovascular and other chronic diseases in humans. Ongoing research from the HVRG connects known functions of the microvasculature that neatly explains its intimate link to disease development in human subjects. Applying clinically relevant data from human tissues, the HVRG team hopes to provide a detailed mechanistic understanding of disease wi the goal to develop novel therape microvascular function. Application of in arterial flow-mediated dilation.

\section{Behind the Research}
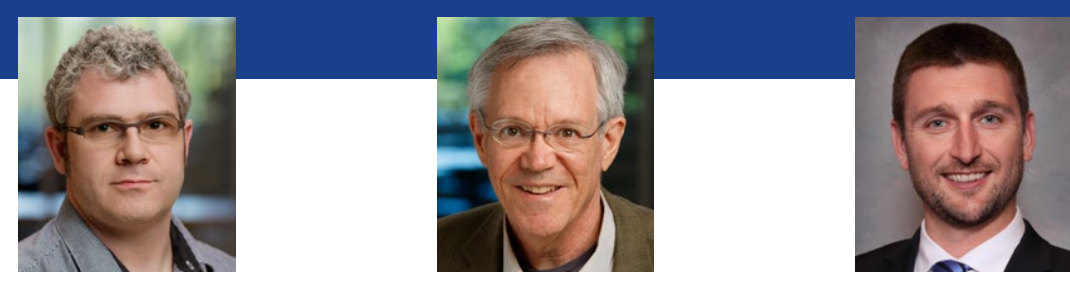

Andreas M. Beyer David D. Gutterman Matthew J. Durand

Julie K. Freed

E: abeyer@mcw.edu T: +14149557514 W: www.mcw.edu/Cardiovascular-Center/Research-Labs/Cardiovascular-Center-FacultyW: www.mcw.edu/departments/pharmacology-and-toxicology/faculty/david-gutterman-md

\section{Research Objectives}

The HVRG studies the complex relationship and physiological effects of vascular stress response to understand how the mechanisms of vasodilation change from nitric oxide (healthy individuals) to mitochondrialderived hydrogen peroxide (individuals with CAD). Severa interconnected signalling pathways including ceramide,
extra-nuclear telomerase, PGCla, LPA, and others have been identified to regulate mitochondrial function in the human microcirculation.

\section{Detail}

Cardiovascular Research Center
Medical College of Wisconsin Medical College of
Milwaukee, W1 53226

MEB 4825, USA

Bio

The HVRG consists of several investigators with diverse backgrounds. Dr David Gutterman is a cardiologist

microcirculation: Dr Andreas Beyer is a trained human and physiologist with a background in vascular function and hypertension research. Dr Julie Freed is a cardiac anesthesiologist with a focus on lipid signalling and vascular function. Dr Matt Durand is a cardiovascular physiologist ischemic conditioning on motor neural, and vascular function in chronic stroke survivors.

Funding Funding
NIH/NHLBI, WE-Care Foundation, Advancing Healthie
Wisconsin, American Heart Association

\section{Collaborators
- Andreas M. Be}

- David D. Gutterman

- Julie K. Freed

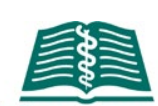

MEDICAL

COLLEGE.

OF WISCONSIN

CARDIOVASCULAR

\section{References}

Gutterman D, Chabowski S, Kadlec A, Durand M, Freed J Gutterman D, Chabowski S, Kadlec A, Durand Mit-Aissa K, Beyer A. (2016). 'The Human Microcirculation' Freed, J.K., et al. (2014). 'Ceramide changes the mediator of flow-induced vasodilation fom nitric oxicle t15(5) p. 525-32.

Beyer, A.M., et al. (2014). 'An acute rise in intraluminal pressure shifts the mediator of flow-mediated dilation from nitric oxide. to hydrogen peroxide in human arterioles'. Am J Physiol Heart

Beyer AM, Freed JK, Durand MJ, Riedel M, Ait-Aissa K, Green P, Hockenberry JC, Morgan RG, Donato AJ, Peleg R, Gasparri M, role for telomerse in the Eriel E, Gutterman DD. (2016). 'Critical' in the human microcirculation. Circ Res, 118(5):856-66.

Durand, M.J., et al. (2015). 'Impaired hyperemic response to exercise post stroke'. PloS one, 10(12): P. e0144023.

\section{Personal Response}

In your research, you make elegant use of human vessels. Can you explain some of the advantages of your methods over animal models?

unique chamanacteristics that have been identified through in vitro studies. Interestingly, dilator responses in commonly use model systems such as rodents, dogs, or pigs do not always human microvessels of critical need. Abnormal microvascular function typically precedes and predicts the development of conduit artery atherosclerosis and its risk factors. This indicates that it may be possible to reverse the negative effects to the developmet of large vessel atherosclerosis, plaques formation, and eventual cardiac events. 